\title{
SPONTANEOUS PERSISTENT CURRENTS IN MESOSCOPIC RINGS
}

\author{
E. ZIPPER AND M. SZOPA
}

Instytut Fizyki, Uniwersytet Śląski, Uniwersytecka 4, 40-007 Katowice, Poland

Persistent currents in mesoscopic systems made of a very clean metal and with nearly flat Fermi surface are studied. It is shown that the inclusion of the orbital magnetic interaction between electrons can lead to spontaneous currents (spontaneous fluxes) and to quantized flux trapping if the number of interacting electrons is large enough. The energy of the system is discussed and the self-consistent formulas for the spontaneous flux and for the quantized flux in the system is derived. The influence of the spin on the presented phenomena is discussed.

PACS numbers: $73.20 . \mathrm{Dx}$

One of the most exciting areas of physics is the study of mesoscopic electronic systems, i.e. metal or semiconductor samples which are sufficiently small and at sufficiently low temperature, such that inelastic electron-phonon scattering is reduced and the electron propagates as a phase coherent wave throughout the entire sample.

If we place the quasi-one-dimensional mesoscopic ring of a radius $R$ in the magnetic field perpendicular to its plane, $B \| \boldsymbol{z}$, the electrons fill the quantized energy levels (for details see [1]):

$$
\varepsilon_{n}=\frac{\hbar^{2}}{2 m R^{2}}\left(n-\frac{\phi}{\phi_{0}}\right)^{2}, \quad n=0, \pm 1, \pm 2, \ldots,
$$

where $n$ parametrizes the orbital angular momentum in $\varphi$ direction, $\phi_{0}=h / e$. To each $\varepsilon_{n}$ there corresponds a current

$$
i_{n} \equiv-\frac{\partial \varepsilon_{n}}{\partial \phi}=\frac{e \hbar}{2 \pi m R^{2}}\left(n-\frac{\phi}{\phi_{0}}\right)
$$

and the total current $I$ is

$$
I(\phi)=\sum_{n} i_{n} f_{\mathrm{FD}}\left(\varepsilon_{n}\right)
$$

where $f_{\mathrm{FD}}\left(\varepsilon_{n}\right)$ is the Fermi-Dirac distribution function.

The system has a set of quantum size energy gaps - the gap at the Fermi surface (FS) is $\Delta=\left(\hbar^{2} / m R^{2}\right) n_{\mathrm{F}}$, where $n_{\mathrm{F}}$ numbers the last occupied state. For the ring with a radius $R=400 \AA$ we get $\Delta \approx 300 \mathrm{~K}$. 
The total energy is periodic in $\phi_{0}$, the current at $k T \ll \Delta$ is persistent because of the gap [1].

The flux $\phi$, which drives the persistent current $I(\phi)$, is the sum of the externally applied flux $\phi_{\mathrm{e}}$ and the flux $\phi_{I}$ from the persistent current itself

$\phi=\phi_{\mathrm{e}}+\phi_{I}$,

where $\phi_{I}=L I$ and $L$ is the self-inductance of the system.

Most theoretical discussions neglect the second term, which is justified by the experimental structures realized so far. Persistent currents driven by the external flux have been predicted by Buttiker, Imry and Landauer [1] and experimentally observed some years later [2].

However, if we consider a very clean system (ballistic regime), made of $M \gg 1$ concentric metallic mesoscopic rings deposited along $z$ axis, the flux $\phi_{I}$ is substantial and cannot be neglected.

Equations (3) and (4) represent then two self-consistent equations for the determination of the current $I$ and the corresponding flux $\phi$. They mean that each electron moves not only in the externally applied flux $\phi_{\mathrm{e}}$ but also in the magnetic flux $\phi_{I}$ produced by all other electrons. Each current loop (channel) is equivalent to the magnetic dipole. The magnetic dipole-dipole interaction is taken here in the mean field approximation. It produces an internal magnetic flux and leads to the nonlinear, implicit equation for the current.

It is also well known $[1,2]$ that the properties of mesoscopic systems depend on the evenness or oddness of the number of electrons in a single ring. Let us assume we consider the system of $M$ mesoscopic rings stacked along $z$ axis, each ring having $N$ electrons.

In case of $N$ odd at $\phi_{\mathrm{e}}=0, T=0$ all states up to $\pm n_{\mathrm{F}}$ are fully occupied. One can calculate the total energy of such system (for details of the calculation see [3])

$$
E(\phi)=E(\phi=0)+\left(\frac{\Delta}{\phi_{0}^{2}}+\frac{1}{2 L}\right) \phi^{2} \geq E(\phi=0), \quad|\phi|<\frac{\phi_{0}}{2} .
$$

This gives $I=0$ in the ground state by pairwise cancellation of the currents in all states.

In case of $N$ even the last two levels $\pm n_{\mathrm{F}}$ below the FS are occupied by a single electron which leads to the spontaneous microscopic current.

The magnetic interaction of these microscopic currents from different rings stacked one above another. along the $z$ axis leads to the magnetically ordered ground state or, in other words, to the spontaneous macroscopic current or spontaneous magnetic flux (for details see [3]).

The total energy in this case is

$$
\begin{gathered}
E(\phi)=E(\phi=0)+\Delta M\left[-\operatorname{sgn} \phi \frac{\phi}{\phi_{0}}+\left(\frac{\phi}{\phi_{0}}\right)^{2}\right]+\frac{\phi^{2}}{2 L} \leq E(\phi=0), \\
|\phi|<\frac{\phi_{0}}{2} .
\end{gathered}
$$

We get then two minima corresponding to spontaneous fluxes

$$
\phi_{\mathrm{SP}}= \pm \frac{\phi_{0}}{2+\gamma}, \quad \text { where } \quad \gamma=\frac{\phi_{0}^{2}}{M \Delta L} .
$$


This flux is approaching $\pm \phi_{0} / 2$ in the limit of small $\gamma$ and zero in the opposite limit. In other words we have a spontaneous orbital current in the ground state

$$
I_{\mathrm{SP}}= \pm \frac{\phi_{0}}{L} \frac{1}{2+\gamma}
$$

which remains persistent also for $0<k T \ll \Delta$.

Let us estimate the average spontaneous flux in case where some of the rings carry an even and some of the rings carry an odd number of electrons. Let us assume that the number of particles on each ring is governed by the Poisson distribution with mean $\bar{N}$ [4]. Performing an average over the number of particles we get an average current

$$
\bar{I}=\frac{M \Delta}{\phi_{0}}\left(\frac{1}{2}-\delta-\frac{|\phi|}{\phi_{0} / 2}\right) \operatorname{sgn} \phi, \quad|\phi|<\frac{\phi_{0}}{2},
$$

where $\delta=\frac{1}{4} \mathrm{e}^{-2 \bar{N}}$.

We see that even for small $\bar{N}$ (e.g. $\bar{N}>10$ ) $\delta \approx 0$. The current (9) is periodic in $\phi_{0} / 2$, i.e. its period is half the period of the persistent current in a single ring.

Equation (9) together with Eq. (4) yield the spontaneous flux

$$
\bar{\phi}_{\mathrm{SP}}= \pm \frac{\phi_{0}}{\left(2+\delta_{1}\right)(2+\gamma)} \text {, }
$$

where

$$
\delta_{1}=\frac{2}{2 \mathrm{e}^{R \bar{N}}-1} .
$$

Here again the factor $\delta_{1}$ can be very well approximated by $\delta_{1} \approx 0$. If the factor $\gamma$ is small ( $M \Delta L$ is large compared to $\left.\phi_{0}^{2}\right)$ then $\phi_{\mathrm{SP}}^{\mathrm{av}} \rightarrow \phi_{0} / 4$. Thus the average spontaneous flux and current are also halved compared to the case where all the rings carry an even number $N$ of electrons. If, for example, $\bar{N}=2 \times 10^{4}, M=10$ and the height of the system of rings is $l=30 \AA$, then with the self-inductance for the cylinder $L=2 \pi \mu_{0} R^{2} / l$, we get $\tilde{\phi}_{\mathrm{SP}}=\phi_{0} / 15$.

In the above calculations we neglected spin. It is to some extent justified in mesoscopic systems because the orbital quantum numbers at the Fermi surface are much larger than spin, and therefore usually one can neglect the contribution of the spin momentum to the total momentum and assume the Hamiltonian to be spin independent.

To take into account spin degrees of freedom, in this paper, we assume that the energy levels $\varepsilon_{n}$ can be occupied by two particles with opposite spin $\sigma=\frac{1}{2},-\frac{1}{2}$. Because the spin energy splitting is very small compared to the orbital splitting we can treat $\sigma$ merely as a label. That kind of analysis has been performed in [4] to investigate field induced currents. The energy and the current are then given by

$$
E^{\sigma}=2 \sum_{n} \varepsilon_{n}, \quad I^{\sigma}=-\frac{\partial E}{\partial \phi} .
$$

We are looking for the spontaneous currents

$$
I_{\mathrm{SP}}^{\sigma}=-\left(\frac{\partial E^{\sigma}}{\partial \phi}\right)_{\phi_{\mathrm{e}}=0}
$$


Assuming that $N=N_{1 / 2}+N_{-1 / 2}$, where $N_{\sigma}$ is the number of particles with spin $\sigma$, we must distinguish four different cases according to the number of particles in each ring.

(1) $N=4 k+1$, where $k$ is an integer

In this case four states $\pm n_{\mathrm{F} \sigma}$ just below the Fermi surface are occupied by 3 electrons and $N_{\sigma}=2 k+1, N_{-\sigma}=2 k$. The current is composed of $I_{\text {odd }}$ for electrons with spin $\sigma$ and $I_{\text {even }}$ for electrons with $-\sigma$. We have

$$
\begin{aligned}
I^{(1)} & =I_{\text {odd }}\left(\frac{N+1}{2}\right)+I_{\text {even }}\left(\frac{N-1}{2}\right) \\
& =\frac{M \Delta}{2 \phi_{0}}\left(1-\frac{2|\phi|}{\phi_{0} / 2}-\frac{1}{N}\right) \operatorname{sgn} \phi, \quad|\phi|<\frac{\phi_{0}}{2} .
\end{aligned}
$$

for $N \gg 1, \frac{1}{N}$ can be neglected and we get the formula for the spontaneous current

$$
I_{\mathrm{SP}}^{(1)}= \pm \frac{\phi_{0}}{2 L} \frac{1}{2+\gamma}
$$

(2) $N=4 k+2$

In this case the four states $\pm n_{\mathrm{F} \sigma}$ are fully occupied and $N_{\sigma}=N_{-\sigma}=$ $2 k+1=N / 2$, i.e. we have an equal, odd number of electrons with $\sigma= \pm \frac{1}{2}$

$$
I^{(2)}=I_{\text {odd }}\left(\frac{N}{2}\right)+I_{\text {even }}\left(\frac{N}{2}\right)=-4 \frac{M \Delta}{\phi_{0}} \frac{\phi}{\phi_{0}}, \quad|\phi|<\frac{\phi_{0}}{2}
$$

and we do not get the spontaneous current

$$
I_{\mathrm{SP}}^{(2)}=0 \text {. }
$$

(3) $N=4 k+3$

In this case the four states $\pm n_{\mathrm{F} \sigma}$ are occupied by one electron and $N_{\sigma}=$ $2(k+1), N_{-\sigma}=2 k+1$. The current is, similarly to the case 1 , composed of $I_{\text {odd }}$ and $I_{\text {even }}$

$$
\begin{aligned}
I^{(3)} & =I_{\text {odd }}\left(\frac{N-1}{2}\right)+I_{\text {even }}\left(\frac{N+1}{2}\right) \\
& =\frac{M \Delta}{2 \phi_{0}}\left(1-\frac{2|\phi|}{\phi_{0 / 2}}+\frac{1}{N}\right) \operatorname{sgn} \phi, \quad|\phi|<\frac{\phi_{0}}{2} .
\end{aligned}
$$

Therefore up to $1 / N, I^{(3)}=I^{(1)}$ and $I_{\mathrm{SP}}^{(3)}=I_{\mathrm{SP}}^{(1)}$.

(4) $N=4 k$

In this case the four states $\pm n_{\mathrm{F} \sigma}$ are occupied by 2 electrons and $N_{\sigma}=$ $N_{\sigma}=2 k$.

We get here

and

$$
I^{(4)}=2 I_{\text {even }}\left(\frac{N}{2}\right)=\frac{M \Delta}{\phi_{0}}\left(1-2 \frac{|\phi|}{\phi_{0}}\right) \operatorname{sgn} \phi, \quad|\phi|<\frac{\phi_{0}}{2}
$$

$$
I_{\mathrm{SP}}^{(4)}= \pm \frac{\phi_{0}}{L} \frac{1}{2+\gamma}
$$


One can observe that in cases (1) and (3) where $N$ is an odd number, the amplitude of the spontaneous current is halved compared to the case (4), where two electrons occupy the last state below the Fermi surface. In case (2) where the last states below the Fermi surface are fully occupied the spontaneous current is zero.

Now we estimate the average spontaneous flux in our spin system. Performing averaging with the Poisson distribution analogous to the one in case of Eqs. (9) and (10) we get the spontaneous flux

$$
\bar{\phi}_{\mathrm{SP}}^{\sigma}=\frac{\phi_{0}}{\left(2+\delta_{1}^{\prime}\right)(2+\gamma)},
$$

where

$$
\delta_{1}^{\prime}=2 \frac{(\bar{N}+1) \sin \bar{N}}{\bar{N} \mathrm{e}^{\bar{N}}-(\bar{N}+1) \sin \bar{N}} .
$$

Here again we see that $\delta_{1}^{\prime} \approx 0$ and the result $(18)$ is effectively equivalent with the spinless case (10). Thus we see that spin effects do not show up in systems which average over a large number of rings.

\section{Discussion}

We have developed a description for the electrons in a system of many quasi-one-dimensional rings deposited along certain axis interacting via the magnetostatic interaction. It is shown that this interaction causes a current carrying ground state by ferromagnetic ordering of the unpaired electrons at the Fermi level.

In other words we get the spontaneous current or the spontaneous magnetic flux in the ground state. The inclusion of spin does not change qualitatively the results and the effect of spin averages to zero in a system of many rings with different, but fixed number of electrons.

The details concerning the behaviour of spontaneous flux at $T>0$ can be found in [3].

\section{Acknowledgment}

The work was supported by grant PB 1770/2/91 of the State Committee for Scientific Research (Republic of Poland).

\section{References}

[1] M. Buttiker, Y. Imry, R. Landauer, Phys. Lett. A 96, 365 (1983); H.F. Cheung, Y. Gefen, E.K. Riedel, W.H. Shih, Phys. Rev. B 37, 6050 (1988); D. Wohlleben, J. Less-Common Met. 138, 11 (1988); M. Szopa, E. Zipper, in: Physics and Materials Science of High Temperature Superconductors, Eds. R. Kossowski, S. Methfessel, D. Wohlleben, NATO ASI Series, Vol. 181, Kluwer, Amsterdam 1990, p. 139.

[2] P. Levy, G. Dolan, J. Dunsmuir, H. Bouchiat, Phys. Rev. Lett. 64, 2074 (1990); V. Chandrasekhar, R.A. Webb, M.J. Brady, M.B. Ketchen, W.J. Gallagher, A. Kleinsasser, Phys. Rev. Lett. 67, 3578 (1991); D. Maily, C. Chapelier, A. Benoit, Phys. Rev. Lett. 70, 2020 (1993). 
[3] M. Szopa, D. Wohlleben, E. Zipper, Phys. Lett. A 160, 271 (1991); D. Wohlleben, M. Esser, P. Freche, E. Zipper, M. Szopa, Phys. Rev. Lett. 66, 3191 (1991); D. Wohlleben, P. Freche, M. Esser, E. Zipper, M. Szopa, Mod. Phys. Lett. B 6, 1481 (1992).

[4] D. Loss, P. Goldbart, Phys. Rev. B 43, 13762 (1991). 